\title{
Five-dimensional cooling and nonlinear dynamics of an optically levitated nanodumbbell
}

\author{
Jaehoon Bang $\odot,{ }^{1}$ T. Seberson, ${ }^{2}$ Peng Ju, ${ }^{2}$ Jonghoon Ahn, ${ }^{1}$ Zhujing Xu, ${ }^{2}$ Xingyu Gao $\odot,{ }^{2}$ \\ F. Robicheaux $(1),{ }^{2,3}$ and Tongcang $\mathrm{Li} \oplus^{1,2,3,4, *}$ \\ ${ }^{1}$ School of Electrical and Computer Engineering, Purdue University, West Lafayette, Indiana 47907, USA \\ ${ }^{2}$ Department of Physics and Astronomy, Purdue University, West Lafayette, Indiana 47907, USA \\ ${ }^{3}$ Purdue Quantum Science and Engineering Institute, Purdue University, West Lafayette, Indiana 47907, USA \\ ${ }^{4}$ Birck Nanotechnology Center, Purdue University, West Lafayette, Indiana 47907, USA
}

(Received 17 April 2020; accepted 16 September 2020; published 9 October 2020)

\begin{abstract}
Optically levitated nonspherical particles in vacuum are excellent candidates for torque sensing, rotational quantum mechanics, high-frequency gravitational wave detection, and multiple other applications. Many potential applications, such as detecting the Casimir torque near a birefringent surface, require simultaneous cooling of both the center-of-mass motion and the torsional vibration (or rotation) of a nonspherical nanoparticle. Here we report five-dimensional cooling of a levitated nanoparticle. We cool the three center-of-mass motion modes and two torsional vibration modes of a levitated nanodumbbell in a linearly polarized laser simultaneously. The only uncooled rigid-body degree of freedom is the rotation of the nanodumbbell around its long axis. This free rotation mode does not couple to the optical tweezers directly. Surprisingly, we observe that it strongly affects the torsional vibrations of the nanodumbbell. This work deepens our understanding of the nonlinear dynamics and rotation coupling of a levitated nanoparticle and paves the way towards full quantum control of its motion.
\end{abstract}

DOI: 10.1103/PhysRevResearch.2.043054

\section{INTRODUCTION}

In recent years, levitated optomechanics provided a fruitful platform for nonequilibrium thermodynamics [1-5], nonlinear dynamics [6-8], precision measurements [9-16], macroscopic quantum mechanics [17-19], and several other applications [20-22]. Besides extensive studies on levitated spherical particles, there is growing interest in levitated nonspherical particles [23-35]. For example, a levitated nanodumbbell in a linearly polarized optical tweezer is an analogy of the Cavendish torsion balance for precision measurements [33]. With a circularly polarized laser, it can rotate at record-high $\mathrm{GHz}$ frequencies [14,33,34,36]. A levitated nanodumbbell has achieved an unprecedented torque sensitivity of $4 \times$ $10^{-27} \mathrm{Nm} / \sqrt{\mathrm{Hz}}$ [14]. Levitated nonspherical particles have also been proposed to measure the Casimir torque [37], create rotational matter-wave interferometers [38], and search for high-frequency gravitational waves [39].

Many potential applications of levitated nonspherical particles require simultaneous cooling of their center-of-mass (c.m.) motions and torsional vibrations (or rotations). For example, to measure the Casimir torque near a birefringent surface with a levitated nanodumbbell or nanorod [37], we will need to cool its c.m. motion to prevent loss near the sur-

\footnotetext{
*tcli@purdue.edu

Published by the American Physical Society under the terms of the Creative Commons Attribution 4.0 International license. Further distribution of this work must maintain attribution to the author(s) and the published article's title, journal citation, and DOI.
}

face, and cool its torsional vibration for detecting the Casimir torque at fixed orientations. To detect the gravitational wave with a levitated microdisk in an optical cavity [39], we will also need to cool its torsional vibrations in addition to c.m. motions to minimize light being scattered out of the optical cavity. Here we report a five-dimensional (5D) cooling of an optically levitated nanodumbbell. We also investigate the nonlinear dynamics of its motion. Surprisingly, we observe that the free rotation of the nanodumbbell around its long axis strongly affects its torsional vibrations. Our work is an essential step towards full quantum control of the rigid-body motion of a levitated nonspherical particle and opens up many potential applications [37-40].

In this work, we investigate the torsional nonlinear dynamics of a silica nanodumbbell levitated in a linearly polarized laser and cool its motion in 5D (Fig. 1). In the focus of a linearly polarized laser, the long axis of the nanodumbbell tends to align with the polarization direction of the laser. Because of the collisions with surrounding air molecules, the nanodumbbell will undergo confined Brownian motion in three translational and two torsional vibration modes, and free Brownian rotation around its long axis. The observed nonlinearity in the torsional motion is much stronger than that in its c.m. motion [6]. The strong nonlinearity can potentially be used for generating nonclassical states and sensing [13,41,42]. Counterintuitively, we also find that the thermal Brownian rotation of the nanodumbbell around its long axis strongly affects its torsional vibrations, even though this rotational mode does not couple to the optical tweezers directly. After investigating the rigid-body dynamics of a levitated nanodumbbell in all six degrees of freedom, we demonstrate 5D cooling of a levitated nanodumbbell. We cool its c.m. motion to a few $\mathrm{K}$ 
(a)

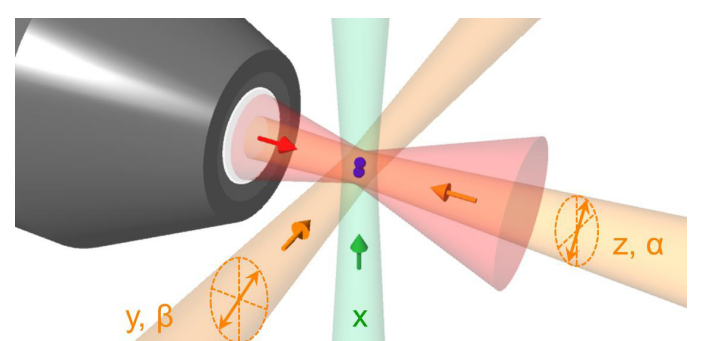

(b)

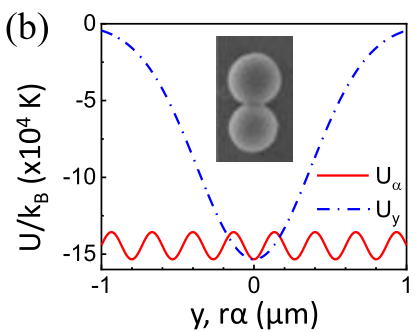

(c)

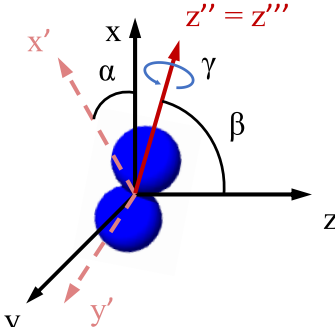

(d)

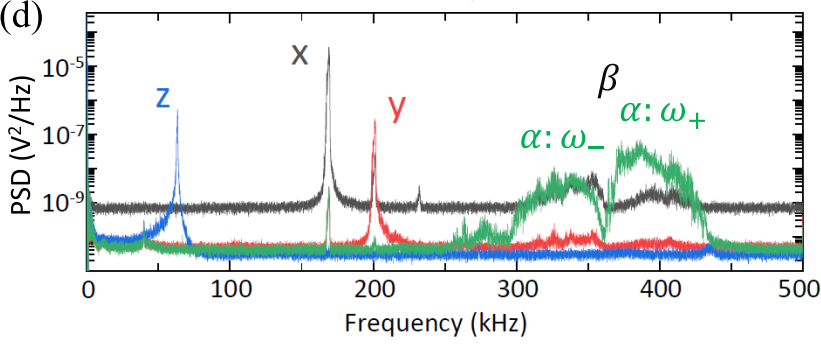

FIG. 1. (a) Schematic of 5D cooling of a levitated nanodumbbell. A 1064-nm laser is tightly focused with an NA $=0.85$ objective lens to levitate a nanodumbbell in vacuum. The 1064-nm trapping laser is polarized in the $x$ direction and propagates along the $z$ axis as indicated by a red arrow. Cooling lasers and their directions of propagation are illustrated in green and orange. The polarization directions of the $y$ and $z$ cooling lasers are tilted to form an angle of about 10 degrees with respect to the polarization direction of the trapping laser. The cooling laser propagating in the $x$ direction is polarized along the $y$ axis. (b) Trapping potential as a function of the c.m. motion along the $y$ axis and the torsional vibration along $\alpha$ direction. For direct comparison, the radius $r=85 \mathrm{~nm}$ is multiplied to the angle $\alpha$ in the torsional potential case. The inset is a scanning electron microscope (SEM) image of a nanodumbbell. (c) Definition of the Euler angles $\alpha, \beta, \gamma$ used in this paper for $z-y^{\prime}-z^{\prime \prime}$ convention. For small libration amplitudes, $\alpha$ represents the motion near the $x$ axis in the $x-y$ plane, and $\beta$ represents the motion in the $x-z$ plane. $\gamma$ stands for the free rotation around the $z^{\prime \prime}=z^{\prime \prime \prime}$ axis. $x^{\prime \prime}, y^{\prime \prime}, x^{\prime \prime \prime}, y^{\prime \prime \prime}$ are not shown to simplify the figure. (d) Power spectral density (PSD) measured for a nanodumbbell, which consists of two 170-nm-diameter silica spheres. PSDs obtained from four different detectors for $x, y, z$, and $\alpha$ are shown. Note that the PSD for the $\beta$ motion could be obtained from the high frequency part of the signal obtained by the $x$ detector. These PSDs are taken at a pressure of $3 \times 10^{-3}$ Torr with $x, y$ and $z$ motion cooling. The data acquisition time is $1 \mathrm{~s}$.

in all three translational directions, and cool its two torsional motions to about $10 \mathrm{~K}$.

\section{NONLINEAR DYNAMICS AND ROTATION COUPLING}

In this experiment, a 1064-nm laser tightly focused by a high NA objective lens $(\mathrm{NA}=0.85)$ is used to trap and de- tect the nanodumbbell in vacuum. The power of the trapping beam at the focus is about $1064 \mathrm{~nm}$. In order to confine the orientation of the nanodumbbell, the trapping laser is linearly polarized along the $x$ axis (Fig. 1). The potential energy of a small nanodumbbell in a linearly polarized Gaussian optical tweezers can be approximately written as

$$
\begin{aligned}
U(\alpha, \beta, x, y, z)= & -\frac{1}{4}\left[\alpha_{\perp}+\left(\alpha_{\|}-\alpha_{\perp}\right) \cos ^{2}(\alpha) \sin ^{2}(\beta)\right] \\
& \times \frac{E_{0}^{2}}{1+\left(\frac{z}{z_{0}}\right)^{2}} \exp \left[-\frac{2 x^{2}}{w_{x}^{2}(z)}-\frac{2 y^{2}}{w_{y}^{2}(z)}\right],
\end{aligned}
$$

where $\alpha_{\|}\left(\alpha_{\perp}\right)$ is the polarizability parallel (perpendicular) to the long axis of the nanodumbbell and $E_{0}$ is the electric field amplitude of the trapping laser at the focus. $w_{x, y}(z)=$ $w_{x, y}(z=0) \cdot \sqrt{1+z^{2} / z_{0}^{2}}$ is the beam waist radius, and $z_{0}$ is the Rayleigh range of the optical tweezers. The nanodumbell is therefore trapped close to the focus and simultaneously aligned parallel to the polarization axis of the trapping laser. The equilibrium orientations are $\alpha=0, \beta=\frac{\pi}{2}$. Note the trapping potential $U(\alpha, \beta, x, y, z)$ is independent of $\gamma$ because of the rotational symmetry of the nanodumbbell [Fig. 1(c)]. The trapping potential as a function of the motion along the $y$ axis $U_{y} \equiv U\left(\alpha=0, \beta=\frac{\pi}{2}, x=0, y, z=0\right)$ and the trapping potential as function of the rotation along the $\alpha$ direction $U_{\alpha} \equiv$ $U\left(\alpha, \beta=\frac{\pi}{2}, x=0, y=0, z=0\right)$ are shown in Fig. 1(b).

The out-going 1064-nm laser beam is collected with a collimation lens after the trap. It is sent to four balanced photodetectors to detect the translational and torsional vibrations. Except the measurement of the torsional vibration in the $\beta$ direction, the optical configurations to detect the motion of the nanodumbbell are similar to those in previous reports $[27,33]$. As shown in Fig. 1(d), the signal obtained from the detector monitoring the translational motion in the $x$ direction contains the information of the torsional motion in the $\beta$ direction. The reason is that the rotation of the nanodumbbell along the $\beta$ direction will deflect the laser beam in the $\beta$ direction, causing a shift of the laser beam along the $x$ axis at the position of the detector [31]. Because the c.m. motion and the torsional vibration have different frequencies, we can separate them with band-pass filters. In this work, we use two different sizes of nanodumbbells. Nanodumbbells consisting of 170-nm-diameter nanospheres are used to study the nonlinear torsional dynamics. Nanodumbbells consisting of two 120-nm-diameter spheres are used for the cooling experiment. Larger nanodumbbells have larger signals, but are more difficult to trap in vacuum with a $1064 \mathrm{~nm}$ laser.

From the measured power spectral densities (PSDs) of a levitated nanodumbbell [Fig. 1(d)], we immediately notice that the torsional peaks $(\alpha$ and $\beta$ ) around $350 \mathrm{kHz}$ are much broader than their expected linear linewidths in vacuum. Thus we need to investigate the nonlinear characteristics of the torsional motion. The c.m. trapping potential of optical tweezers can be approximated by a Gaussian potential, which is not harmonic. This causes nonlinear translational motion of a trapped nanoparticle in vacuum [6,43]. For torsional vibrations, the nonlinearity is much stronger because the potential $U(\alpha, \beta, x, y, z)$ is sinusoidally dependent on $\alpha$ and $\beta$ [Fig. 1(b)]. The trapping depth for the orientation confinement is also much smaller than that for the c.m. confinements 
(a)

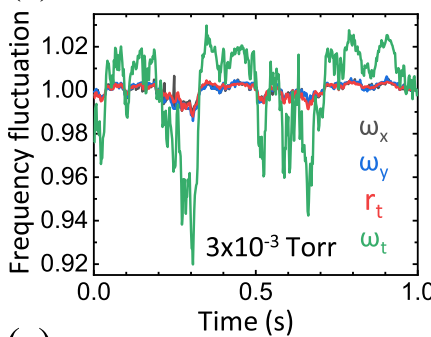

(c)

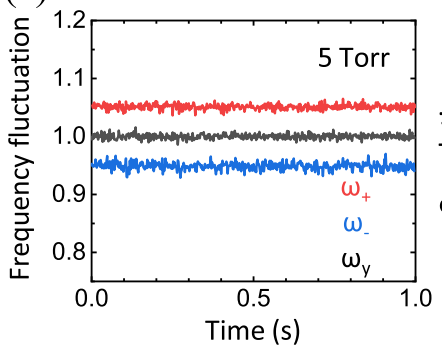

(b)

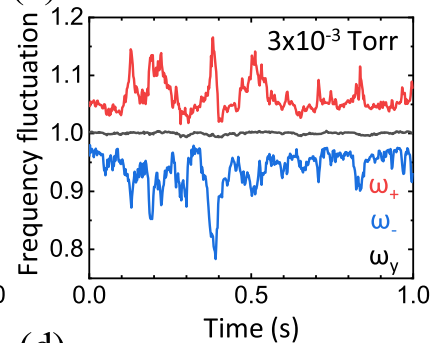

(d)

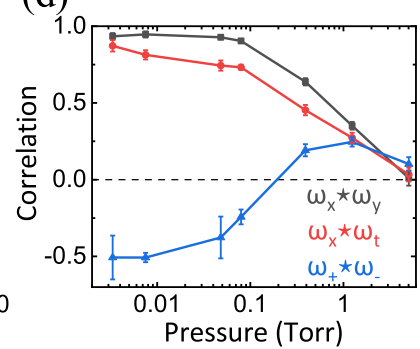

(a)

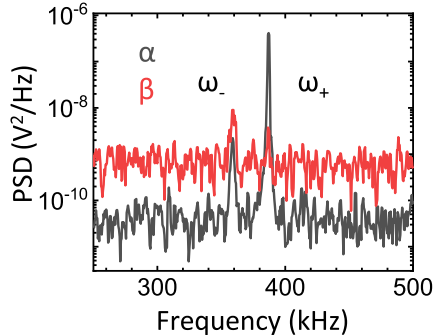

(c)

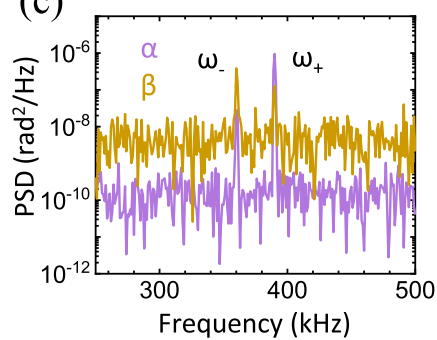

(b)

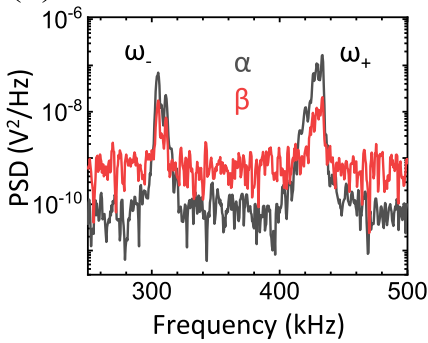

(d)

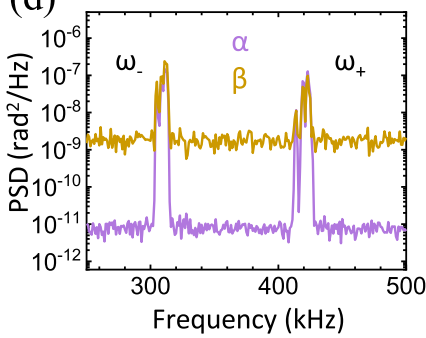

FIG. 2. (a) Relative frequency fluctuations for translational motions $\left(\omega_{x}, \omega_{y}\right)$ and torsional motions at $3.33 \times 10^{-3}$ Torr. (b) Relative frequency fluctuations of $\omega_{+}$(red), $\omega_{-}$(blue), and $\omega_{y}$ (grey) at $3.33 \times 10^{-3}$ Torr. The $\omega_{+}$and $\omega_{-}$frequencies are normalized by the average $\left\langle\left(\omega_{+}+\omega_{-}\right) / 2\right\rangle$. $\omega_{y}$ is normalized by its average $\left\langle\omega_{y}\right\rangle$. (c) Relative frequency fluctuations of $\omega_{+}$(red), $\omega_{-}$(blue), $\omega_{y}$ (grey) at 5 Torr. In (a), (b), (c), each frequency is determined from a PSD corresponding to $2 \mathrm{~ms}$ of data in time. (d) Frequency correlations between different degrees of freedom. The correlations are plotted as a function of pressure. Each data point corresponds to an average of five sets of measurements. For each measurement, the correlations are calculated from 500 PSD data. The error bar shows the standard deviation.

[Fig. 1(b)]. To simplify the problem, we apply feedback cooling to c.m. motions so the nonlinearity caused by the thermal c.m. motion can be neglected. We introduce the deviation angles from the equilibrium orientations as $\xi=\alpha, \eta=\frac{\pi}{2}-$ $\beta$. If the deviation angles are small, we can approximate the system as a Duffing nonlinear oscillator. The nonlinear c.m. motion frequencies $\left(\omega_{x_{i}}, x_{i}=x, y, z\right)$ and torsional frequencies $\left(\omega_{\xi}, \omega_{\eta}\right)$ can be obtained from Eq. (1):

$$
\begin{aligned}
\omega_{x_{i}}^{2} & =\omega_{x_{i}, 0}^{2}\left[1-\frac{\alpha_{\|}-\alpha_{\perp}}{\alpha_{\|}}\left(\xi^{2}+\eta^{2}\right)\right], \\
\omega_{\xi}^{2} & =\omega_{\xi, 0}^{2}\left(1-\frac{2}{3} \xi^{2}-\eta^{2}\right), \\
\omega_{\eta}^{2} & =\omega_{\eta, 0}^{2}\left(1-\xi^{2}-\frac{2}{3} \eta^{2}\right) .
\end{aligned}
$$

Here $\omega_{x_{i}, 0}, \omega_{\xi, 0}, \omega_{\eta, 0}$ are intrinsic trapping frequencies when the vibration amplitudes are 0. Based on Eq. (2), a finite-amplitude vibration in any direction will decrease the frequencies in all modes simultaneously. Thus we expect the frequency fluctuations of all modes to be positively correlated.

The measured frequency fluctuations are presented in Fig. 2. When the pressure is relatively high (5 Torr), frequency fluctuations of the two modes are small because of the high damping rate [Fig. 2(c)]. When the pressure decreases, however, the rarefied surrounding gas is unable to provide enough

FIG. 3. (a), (b) Measured PSDs of motions along $\alpha$ (grey) and $\beta$ (red) directions when $\omega_{c}$ is small (a) or large (b). The PSDs are taken at $3 \times 10^{-3}$ Torr and the measurement time is $2 \mathrm{~ms}$. (c), (d) Corresponding simulation results for $\alpha$ (purple) and $\beta$ (yellow) motions when $\omega_{c} / 2 \pi$ is $17 \mathrm{kHz}$ (c) or $115 \mathrm{kHz}(\mathrm{d})$. The simulated motion time is $1 \mathrm{~ms}$. Random background noise is added to mimic the experiment.

damping during the measurement time $(2 \mathrm{~ms})$. As shown in Fig. 2(a), the thermal motion then causes large frequency fluctuations because of the nonlinearity. Besides the large frequency fluctuations, another consequence of nonlinearity is the strong correlation of frequency fluctuations in different modes. As expected from Eq. (2), the relative fluctuations in c.m. frequencies $\omega_{x_{i}} /\left\langle\omega_{x_{i}}\right\rangle$ at $3 \times 10^{-3}$ Torr are positively correlated [Fig. 2(a)]. The normalized correlation of the c.m. frequency fluctuations $\omega_{x} \star \omega_{y}$ becomes close to one (0.93 \pm 0.02 at $3 \times 10^{-3}$ Torr) as the pressure decreases [Fig. 2(d)], which is similar to the case for a levitated single nanosphere [6]. To further test Eq. (2), we introduce $\omega_{t} \equiv \sqrt{\left(\omega_{\xi}^{2}+\omega_{\eta}^{2}\right) / 2}$, and its adjusted relative fluctuation $r_{t}=1+\frac{6}{5} \frac{\alpha_{\Perp} \alpha_{\perp}}{\alpha_{\|}}\left(\frac{\omega_{t}}{\left\langle\omega_{t}\right\rangle}-\right.$ 1). For a nanodumbbell with an aspect ratio of 1.9 , we have $\frac{\alpha_{\|}-\alpha_{\perp}}{\alpha_{\|}}=0.126$ [33]. The adjusted relative fluctuation $r_{t}$ in torsional frequencies is plotted together with fluctuations in c.m. frequencies in Fig. 2(a). $r_{t}$ overlaps with $\omega_{x_{i}} /\left\langle\omega_{x_{i}}\right\rangle$ very well, which agrees with Eq. (2).

However, Eq. (2) could not explain all features in the measured torsional PSDs. For example, the measured torsional PSDs have two peaks for both $\alpha$ and $\beta$ motions [Fig. 1(d)]. The frequencies of these two peaks $\left(\omega_{+}, \omega_{-}\right)$are negatively correlated at low pressure [Fig. 2(b)], which could not be explained by Eq. (2). This disagreement is because we have not considered the free rotation of the nanodummbell around its long axis. This rotation will couple the two torsional modes [31]: $\ddot{\xi}=-\omega_{\xi}^{2} \xi-\omega_{c} \dot{\eta}, \ddot{\eta}=-\omega_{\eta}^{2} \eta+\omega_{c} \dot{\xi}$. Here $\omega_{c}=$ $\left(I_{z} / I_{x}\right) \omega_{\gamma}$ and $\omega_{\gamma}$ is the angular frequency of its spin around its symmetric axis. Because of the rotation coupling, the solutions for $\xi$ and $\eta$ have two normal modes $\omega_{+}$and $\omega_{-}$, which can be understood as clockwise and counterclockwise precession modes. They are hybrid modes of the torsional 
(a)

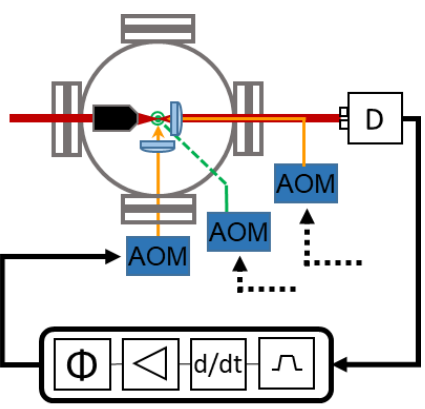

(d)

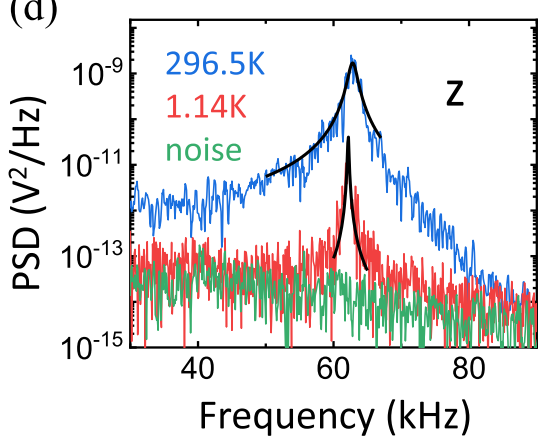

(b)

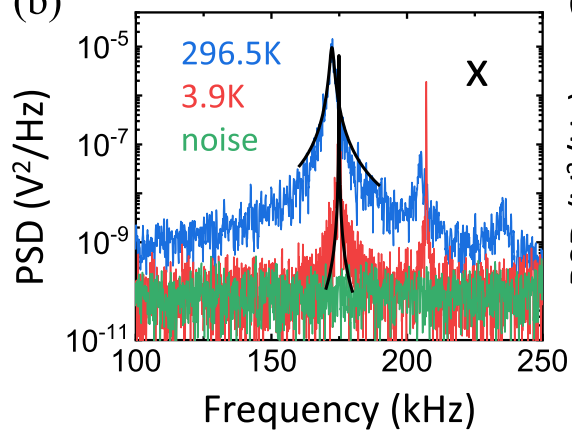

(e)

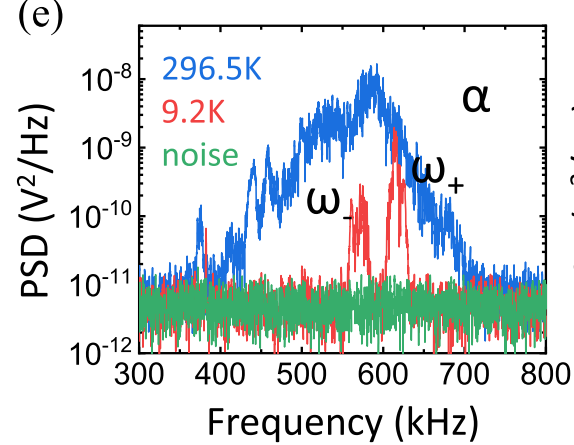

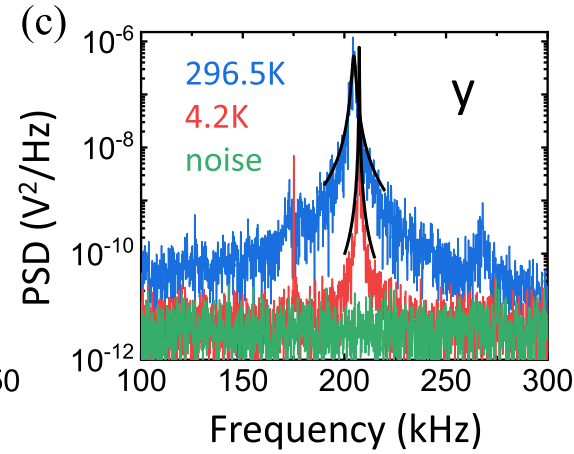

(f) $10^{-11}$

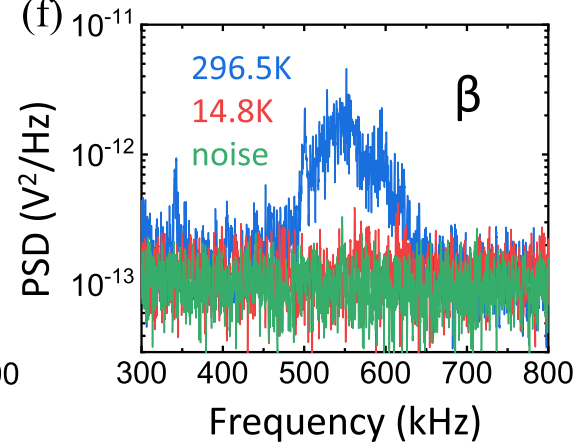

FIG. 4. 5D cooling of a levitated nanodumbbell. (a) Simplified schematic of the setup showing the sequence of cooling for a single direction. In the real experiment, signals from the particle are collected with four balanced detectors (D) and processed with five home-built circuits to generate the corresponding feedback signals. The signal obtained from the balanced detector for both $x$ and $\beta$ motions is split to two and fed into two different derivative circuits in order to generate the cooling signals for the motion in $x$ and $\beta$ directions. After processing, signals corresponding to $\alpha$ and $\beta$ motions are added to the signals for $z$ and $y$ motions, respectively [Fig. 1(a)]. These signals are then used to modulate the cooling lasers. The results of 5D feedback cooling are shown in (b) $x$, (c) $y$, (d) $z$, (e) $\alpha$, and (f) $\beta$. The blue curves are PSDs before cooling, and the red curves are PSDs with cooling. The green spectra show the noise levels when there is no particle. The pressure is 1 Torr for blue curves (no cooling), and is $1.8 \times 10^{-3}$ Torr for red curves (with cooling). The effective temperatures for $x, y$, and $z$ motions are calculated based on Lorentzian fittings (shown in grey dashed lines). The effective temperatures for $\alpha$ and $\beta$ motions are calculated by comparing the areas below the PSDs.

motions. We have

$$
\omega_{ \pm}=\frac{1}{\sqrt{2}}\left[2 \omega_{t}^{2}+\omega_{c}^{2} \pm \sqrt{4 \omega_{t}^{2} \omega_{c}^{2}+\omega_{c}^{4}+\Delta^{4}}\right]^{\frac{1}{2}},
$$

where $\Delta^{2}=\omega_{\xi}^{2}-\omega_{\eta}^{2}$. If $\Delta^{4}<<4 \omega_{t}^{4}$, which is the case in our experiment, we have $\omega_{t}=\sqrt{\omega_{+} \omega_{-}}$. This equation is used to calculate $\omega_{t}$ shown in Fig. 2. $\omega_{+}$and $\omega_{-}$change oppositely when $\omega_{c}$ changes. Due to the Brownian rotation of the nanodumbbell, the two torsional peaks are enforced to move in the opposite direction as can be seen in Fig. 2(b). This competes with the nonlinear effect [Eq. (2)] and eventually becomes superior as the pressure goes down. As a result, the correlation between the frequencies of these two hybrid modes $\omega_{+} \star \omega_{-}$is negative in high vacuum [Fig. 2(d)].

To further investigate the nature of complex rigid-body motion of a levitated nanodumbbell, we perform numerical simulations of the 6D Brownian motion of a nanodumbbell. Examples of simulation results and experimental results of the PSDs of torsional vibrations are shown in Fig. 3. As shown in the PSD plot, the frequency difference of the two hybrid modes $\omega_{+}$and $\omega_{-}$becomes smaller when the coupling frequency $\left(\omega_{c}\right)$ decreases. Note that $\omega_{c}$ is determined by the geometry of the particle $\left(I_{z} / I_{x}\right)$ and Brownian rotation frequency $\omega_{\gamma}$. This observation means that even though the spin of the nanodumbbell around its symmetric axis does not directly interact with the trapping laser, its angular velocity $\left(\omega_{\gamma}\right)$ can be monitored by tracking the frequency separation of the two hybrid modes. This result paves a way towards getting access to this "invisible" degree of freedom that does not couple to the optical tweezer directly.

\section{5D COOLING}

To cool the translational and torsional vibrations of a nanodumbbell, we apply three linearly polarized cooling lasers along $x, y$ and $z$ directions as illustrated in Fig. 1(a). The wavelength of the $x$ cooling laser is $532 \mathrm{~nm}$, and the wavelengths of the $y$ and $z$ cooling lasers are $976 \mathrm{~nm}$. To avoid interference between the $y$ and $z$ cooling lasers, the $976 \mathrm{~nm}$ laser has a short coherence length. The intensities of the $x$ and $y$ cooling lasers are both roughly $1 \mathrm{~mW} / \mu \mathrm{m}^{2}$, and the intensity of the $z$ cooling laser is roughly $5 \mathrm{~mW} / \mu \mathrm{m}^{2}$. The scattering forces from the three cooling lasers are used to cool the nanodumbbell's c.m. motions. To cool the torsional vibrations, we intentionally tilt the polarization axes of the $y$ and $z$ cooling lasers by about 10 degrees with respect to the direction of polarization of the trapping laser. Thus the $z$ cooling laser can exert a torque on the nanodumbell to cool its $\alpha$ torsional mode, and the $y$ cooling laser can exert a torque to cool its $\beta$ torsional vibration mode. The polar- 
ization direction of the $x$ cooling laser is kept to be parallel to the $y$ axis and it is not used to cool any torsional degree of freedom.

As shown in Fig. 4(a), signals about the translational and torsional vibrations of the nanodumbbell are sent to electronic circuits to control the powers of the three cooling lasers with acousto-optic modulators (AOMs). We use five home-built circuits with bandpass filters, differentiators, and variable gain amplifiers to process the signals for cooling. We use a bandpass filter with a frequency range of $220 \mathrm{kHz}-5 \mathrm{MHz}$ to obtain the $\beta$ signal from the output of the $x$ detector [Fig. 1(d)]. The differentiators calculate the derivatives of the motional signals and provide velocity information for cooling. The two torsional cooling signals for $\alpha$ and $\beta$ motions are added on top of the translational cooling signals for $z$ and $y$ motions using adder circuits before feeding into the AOM drivers. The powers of the cooling lasers are modulated as $\Delta P_{x}=-C_{x} \frac{d x}{d t}$, $\Delta P_{y}=-C_{y} \frac{d y}{d t}-C_{\beta} \frac{d \beta}{d t}$, and $\Delta P_{z}=-C_{z} \frac{d z}{d t}-C_{\alpha} \frac{d \alpha}{d t}$ to achieve $5 \mathrm{D}$ cooling. Here $C_{x}, C_{y}, C_{z}, C_{\alpha}$, and $C_{\beta}$ are modulation coefficients controlled by variable gain amplifiers.

The results of 5D cooling of a levitated nanodumbbell are shown in Fig. 4. Changes of the PSDs due to feedback cooling are plotted for each degree of freedom. The c.m. motions of the nanodumbbell are cooled to a few $\mathrm{K}$ at $1.8 \times 10^{-3}$ torr in all three directions. The energy of the two torsional modes are also reduced by feedback cooling. Since both hybrid modes $\left(\omega_{+}, \omega_{-}\right)$contribute to the torsional motions along $\alpha$ and $\beta$ directions, we consider both peaks together to extract the motional temperature. The lowest effective temperatures achieved for the two torsional degrees of freedom are $9.2 \mathrm{~K}$ $(\alpha)$ and $14.8 \mathrm{~K}(\beta)$, respectively. This is mainly limited by the low signal-to-noise ratio of the $\beta$ signal obtained from the $x$ detector. In fact, the PSD of the cooled $\beta$ vibration is close to its noise level [Fig. 4(f)]. In the future, we can add another laser along the $y$ axis to detect this mode more efficiently. The nanodumbbell has six motional degrees of freedom in total.
The uncooled degree of freedom, which is the spin motion $(\gamma)$, does not directly interact with the laser because of the symmetry of the nanodumbbell.

\section{CONCLUSION}

In conclusion, we investigate the nonlinear dynamics of a levitated nanodumbbell and demonstrate 5D cooling of its motion. The frequency fluctuations of the torsional motions are observed to be much larger than those of the c.m. motions. In the case of the torsional motions, it turns out that the two peaks in the frequency domain are influenced by two distinct factors: nonlinearity and rotation coupling. The large nonlinearity of the torsional motion could be advantageous for creating nonclassical states and sensing $[13,41,42]$. We also demonstrate 5D cooling of a levitated nanodumbbell by developing an active force and torque feedback cooling method. The only uncooled degree of freedom is the spin around its long axis, which has no direct interaction with the trapping laser. We could successively observe the effect of this rotational degree of freedom via the relative frequency difference of the two hybrid modes of the torsional motions. Thus, the angular frequency of the nanodumbbell's rotation can be observed even if the rotational motion itself is not observable. This work helps us to better understand the dynamics of a levitated nonspherical particle and cool its motion to the ground state in all degrees of freedom in the future. Our work is also relevant to cooling of other nonsphereric particles, such as nanorods [38], microdisks [24,39], and mirrors [40] for exploring new physics.

\section{ACKNOWLEDGMENTS}

We are grateful for the support from the Office of Naval Research under Grant No. N00014-18-1-2371 and the NSF under Grant No. PHY-1555035.
[1] T. Li, S. Kheifets, D. Medellin, and M. G. Raizen, Science 328, 1673 (2010).

[2] J. Gieseler, R. Quidant, C. Dellago, and L. Novotny, Nat. Nanotechnol. 9, 358 (2014).

[3] J. Millen, T. Deesuwan, P. Barker, and J. Anders, Nat. Nanotechnol. 9, 425 (2014).

[4] T. M. Hoang, R. Pan, J. Ahn, J. Bang, H. T. Quan, and T. Li, Phys. Rev. Lett. 120, 080602 (2018).

[5] S. Liu, Z.-q. Yin, and T. Li, Adv. Quant. Technol. 3, 1900099 (2020).

[6] J. Gieseler, L. Novotny, and R. Quidant, Nat. Phys. 9, 806 (2013).

[7] P. Z. G. Fonseca, E. B. Aranas, J. Millen, T. S. Monteiro, and P. F. Barker, Phys. Rev. Lett. 117, 173602 (2016).

[8] Y. Arita, S. H. Simpson, P. Zemánek, and K. Dholakia, Sci. Adv. 6, eaaz9858 (2020)

[9] A. A. Geraci, S. B. Papp, and J. Kitching, Phys. Rev. Lett. 105, 101101 (2010).

[10] Y. Arita, M. Mazilu, and K. Dholakia, Nat. Commun. 4, 2374 (2013).
[11] D. C. Moore, A. D. Rider, and G. Gratta, Phys. Rev. Lett. 113, 251801 (2014).

[12] G. Ranjit, M. Cunningham, K. Casey, and A. A. Geraci, Phys. Rev. A 93, 053801 (2016).

[13] S. Qvarfort, A. Serafini, P. F. Barker, and S. Bose, Nat. Commun. 9, 3690 (2018).

[14] J. Ahn, Z. Xu, J. Bang, P. Ju, X. Gao, and T. Li, Nat Nanotechnol. 15, 89 (2020).

[15] D. Carney, G. Krnjaic, D. C. Moore, C. A. Regal, G. Afek, S. Bhave, B. Brubaker, T. Corbitt, J. Cripe, N. Crisosto, A. Geraci, S. Ghosh, J. G. E. Harris, A. Hook, E. W. Kolb, J. Kunjummen, R. F. Lang, T. Li, T. Lin, Z. Liu, J. Lykken, L. Magrini, J. Manley, N. Matsumoto, A. Monte, F. Monteiro, T. Purdy, C. J. Riedel, R. Singh, S. Singh, K. Sinha, J. M. Taylor, J. Qin, D. J. Wilson, and Y. Zhao, Mechanical quantum sensing in the search for dark matter, arXiv:2008.06074 (2020).

[16] Y. Zheng, L.-M. Zhou, Y. Dong, C.-W. Qiu, X.-D. Chen, G.-C. Guo, and F.-W. Sun, Phys. Rev. Lett. 124, 223603 (2020).

[17] D. E. Chang, C. A. Regal, S. B. Papp, D. J. Wilson, J. Ye, O. Painter, H. J. Kimble, and P. Zoller, Proc. Nat. Acad. Sci. 107, 1005 (2010). 
[18] U. Delić, M. Reisenbauer, K. Dare, D. Grass, V. Vuletić, N. Kiesel, and M. Aspelmeyer, Science 367, 892 (2020).

[19] S. Bose, A. Mazumdar, G. W. Morley, H. Ulbricht, M. Toroš, M. Paternostro, A. A. Geraci, P. F. Barker, M. S. Kim, and G. Milburn, Phys. Rev. Lett. 119, 240401 (2017).

[20] Z.-Q. Yin, A. A. Geraci, and T. Li, Int. J. Mod. Phys. B 27, 1330018 (2013).

[21] J. Millen, T. S. Monteiro, R. Pettit, and A. N. Vamivakas, Rep. Prog. Phys. 83, 026401 (2020).

[22] Y. Huang, Q. Guo, A. Xiong, T. Li, and Z.-q. Yin, Phys. Rev. A 102, 023113 (2020).

[23] O. Romero-Isart, M. L. Juan, R. Quidant, and J. I. Cirac, New J. Phys. 12, 033015 (2010).

[24] S. Singh, G. A. Phelps, D. S. Goldbaum, E. M. Wright, and P. Meystre, Phys. Rev. Lett. 105, 213602 (2010).

[25] H. Shi and M. Bhattacharya, J. Mod. Opt. 60, 382 (2013).

[26] W. Lechner, S. J. M. Habraken, N. Kiesel, M. Aspelmeyer, and P. Zoller, Phys. Rev. Lett. 110, 143604 (2013).

[27] T. M. Hoang, Y. Ma, J. Ahn, J. Bang, F. Robicheaux, Z.-Q. Yin, and T. Li, Phys. Rev. Lett. 117, 123604 (2016).

[28] S. Kuhn, A. Kosloff, B. A. Stickler, F. Patolsky, K. Hornberger, M. Arndt, and J. Millen, Optica 4, 356 (2017).

[29] B. A. Stickler, B. Schrinski, and K. Hornberger, Phys. Rev. Lett. 121, 040401 (2018).

[30] M. Rashid, M. Toroš, A. Setter, and H. Ulbricht, Phys. Rev. Lett. 121, 253601 (2018).
[31] T. Seberson and F. Robicheaux, Phys. Rev. A 99, 013821 (2019).

[32] T. Delord, P. Huillery, L. Nicolas, and G. Hétet, Nature (London) 580, 56 (2020).

[33] J. Ahn, Z. Xu, J. Bang, Y.-H. Deng, T. M. Hoang, Q. Han, R.-M. Ma, and T. Li, Phys. Rev. Lett. 121, 033603 (2018).

[34] F. van der Laan, R. Reimann, A. Militaru, F. Tebbenjohanns, D. Windey, M. Frimmer, and L. Novotny, Phys. Rev. A 102, 013505 (2020).

[35] Y. Ma, K. E. Khosla, B. A. Stickler, and M. S. Kim, Phys. Rev. Lett. 125, 053604 (2020).

[36] R. Reimann, M. Doderer, E. Hebestreit, R. Diehl, M. Frimmer, D. Windey, F. Tebbenjohanns, and L. Novotny, Phys. Rev. Lett. 121, 033602 (2018).

[37] Z. Xu and T. Li, Phys. Rev. A 96, 033843 (2017).

[38] B. A. Stickler, B. Papendell, S. Kuhn, B. Schrinski, J. Millen, M. Arndt, and K. Hornberger, New J. Phys. 20, 122001 (2018).

[39] A. Arvanitaki and A. A. Geraci, Phys. Rev. Lett. 110, 071105 (2013).

[40] G. Guccione, M. Hosseini, S. Adlong, M. T. Johnsson, J. Hope, B. C. Buchler, and P. K. Lam, Phys. Rev. Lett. 111, 183001 (2013).

[41] M. Aspelmeyer, T. J. Kippenberg, and F. Marquardt, Rev. Mod. Phys. 86, 1391 (2014).

[42] R. L. Badzey and P. Mohanty, Nature (London) 437, 995 (2005).

[43] M. Yoneda and K. Aikawa, J. Phys. B. 50, 245501 (2017). 